\title{
Lisocabtagene Maraleucel
}

National Cancer Institute

\section{Source}

National Cancer Institute. Lisocabtagene Maraleucel. NCI Thesaurus. Code C125192.

A preparation of a defined ratio of CD4+ and CD8+ autologous T-lymphocytes transduced with a lentiviral vector expressing a chimeric antigen receptor (CAR) containing an anti-CD19 single chain variable fragment (scFv) fused to the signaling domain of 4-1BB (CD137), the zeta chain of the TCR/CD3 complex (CD3-zeta), and a truncated form of the human epidermal growth factor receptor (EGFRt), with potential immunostimulating and antineoplastic activities. Upon intravenous administration, lisocabtagene maraleucel is directed to and induces selective toxicity in CD19-expressing tumor cells. CD19 antigen is a B-cell specific cell surface antigen expressed in all B-cell lineage malignancies. Devoid of both ligand binding domains and tyrosine kinase activity, the expressed EGFRt both facilitates in vivo detection of the administered, transduced Tcells and can promote elimination of those cells through a cetuximab-induced antibody dependent cellular cytotoxicity (ADCC) response. The 4-1BB costimulatory signaling domain enhances both proliferation of T-cells and antitumor activity. 